\title{
Atrioventricular and Sinus Node Dysfunction in Stable COVID-19 Patients
}

\author{
Savalan Babapoor-Farrokhran ${ }^{1} \cdot$ Uyanga Batnyam ${ }^{1}$ • Philip C. Wiener ${ }^{1} \cdot$ Napatt Kanjanahattakij $^{1} \cdot$ Ola Khraisha $^{1}$. \\ Aman Amanullah ${ }^{1,2}$. Sumeet K. Mainigi ${ }^{1,2}$
}

Accepted: 28 August 2020 / Published online: 4 September 2020

(C) Springer Nature Switzerland AG 2020

\begin{abstract}
There are now well-documented cardiac complications of COVID-19 infection which include myocarditis, heart failure, and acute coronary syndrome resulting from coronary artery thrombosis or SARS-CoV-2-related plaque ruptures. There is growing evidence showing that arrhythmias are also one of the major complications. We report two patients with no known history of cardiac conduction disease who presented with COVID-19 symptoms, positive SARS-CoV-2 infection, and developed cardiac conduction abnormalities. Cardiac conduction system disease involving the sino-atrial (SA) node and atrioventricular (AV) node could be a manifestation of SARS-CoV-2 infection.
\end{abstract}

Keywords Sinus node dysfunction $\cdot$ Atrioventricular block $\cdot$ Bradycardia $\cdot$ COVID-19 $\cdot$ Arrhythmia

\section{Introduction}

There are now well-documented cardiac complications of COVID-19 infection which include myocarditis, heart failure, and acute coronary syndrome resulting from coronary artery thrombosis or SARS-CoV-2-related plaque ruptures [1, 2]. However, there is growing evidence showing that arrhythmias are also one of the major complications. Liu et al. reported that about $7 \%$ of patients report palpitations as a presenting symptom [3]. In a recent report from Wuhan, China, $16.7 \%$ of hospitalized and $44.4 \%$ of ICU patients with COVID-19 had cardiac arrhythmias [4]. To date, there have not been reports of reversible sinus node and atrioventricular nodal dysfunction associated with COVID-19 in stable patients. This case series describes clinical characteristics of COVID-19 patients who presented with reversible bradycardia.

This article is part of the Topical Collection on COVID-19

Savalan Babapoor-Farrokhran

Babapoos@einstein.edu

1 Division of Cardiology, Department of Medicine, Einstein Medical Center, 5501 Old York Road, Philadelphia, PA 19141, USA

2 Sidney Kimmel Medical College, Thomas Jefferson University, Philadelphia, PA 19107, USA

\section{Case Presentation}

\section{Patient 1}

A 69-year-old woman with a history of hypertension, hyperlipidemia, diabetes mellitus type 2, cerebrovascular accident, and asthma who presented to our institution with symptoms of headaches, shortness of breath, cough, sweating, and generalized body aches. Initial vital signs were an oral temperature of $36.8^{\circ} \mathrm{C}\left(98.6^{\circ} \mathrm{F}\right)$, a heart rate of 54 beats per minute (bpm), a blood pressure of $220 / 96 \mathrm{mmHg}$, and a respiratory rate of 24 breaths per minute. Significant lab work included positive reverse transcriptase polymerase chain reaction (RT-PCR) testing for SARS-CoV-2. Electrolytes including potassium and magnesium were within normal limits. Renal function and troponin-I were normal. Due to her hypertensive urgency, she was treated briefly with nicardipine infusion and transitioned to oral amlodipine and hydralazine. She was otherwise stable and did not receive any specific treatment for COVID-19 infection. Transthoracic echocardiography revealed normal biventricular systolic function with left ventricular ejection fraction of (LVEF) $>65 \%$, normal diastolic function, and without valvular pathology. Initial electrocardiogram (ECG) demonstrated a heart rate of $78 \mathrm{bpm}$, normal sinus rhythm with sinus arrhythmia, non-specific T-wave abnormality, and prolonged QTc of $474 \mathrm{~ms}$ (Fig. 1a). She subsequently developed sinus bradycardia with a 2:1 atrioventricular (AV) 
a

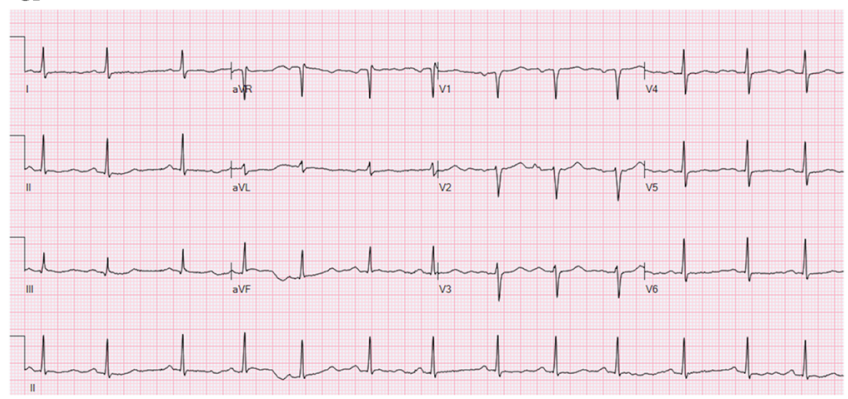

C



Fig. 1 Representative electrocardiogram and telemetry tracings of the patient in case 1. a Admission ECG demonstrating heart rate of $78 \mathrm{bpm}$, normal sinus rhythm with sinus arrhythmia, non-specific $T$ wave abnormality, prolonged QTc of $474 \mathrm{~ms}$. b Electrocardiogram demonstrating sinus bradycardia heart rate of $46 \mathrm{bpm}$ with $2: 1 \mathrm{AV}$ block, and non-

block and heart rate around 40 bpm (Fig. 1b). In addition, cardiac telemetry monitoring revealed intermittent 2:1 AV block through the 8th day of admission (Fig. 1b). Otherwise, she remained asymptomatic from cardiac standpoint throughout hospitalization and her respiratory status was unchanged and treated conservatively. Her conduction abnormality resolved by day 13 of admission, and subsequent serial ECGs did not demonstrate any further evidence of AV block (Fig. 1d). She was subsequently discharged in stable condition. Differential diagnosis of the underlying causes of bradycardia and transient sinus arrest in this case includes SARS-CoV2 infection, myocardial ischemia/infarction, myocarditis, cardiomyopathies, hypoxia, electrolyte disturbances, medication toxicity (e.g., calcium channel blockers, beta blockers), and increased vagal tone. She was not on negative ionotropic agents before admission and was only taking amlodipine for hypertension. SARS-CoV2 infection is highly likely due to the absence of convincing evidence suggesting other etiologies.

\section{Patient 2}

An 83-year-old woman with a history of hypertension, and hyperlipidemia, presented to our hospital with shortness of breath, nasal congestion, and diarrhea. Initial vital signs were an oral temperature of $37.0{ }^{\circ} \mathrm{C}\left(98.6{ }^{\circ} \mathrm{F}\right)$, a heart rate of $88 \mathrm{bpm}$, a blood pressure of 203/90 $\mathrm{mmHg}$, and a respiratory b

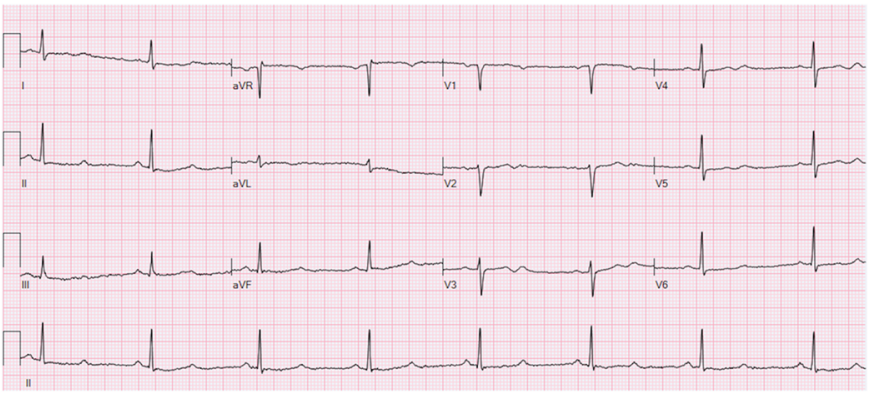

d

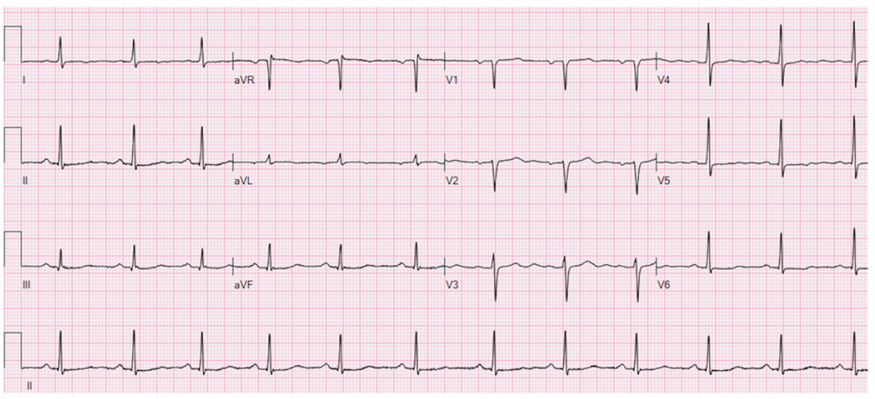

specific $\mathrm{T}$ wave abnormality. c Telemetry tracing of the patient demonstrating sinus bradycardia and 2:1 AV block. d ECG demonstrating normal sinus rhythm, non-specific T wave abnormality, prolonged QTc of $454 \mathrm{~ms}$

rate of $20 \mathrm{br} / \mathrm{min}$. She tested positive for COVID-19 using RT-PCR. Renal function and electrolytes including potassium and magnesium were normal. Troponin-I was $0.76 \mathrm{ng} / \mathrm{mL}$ $(0.0-0.03 \mathrm{ng} / \mathrm{mL})$. Transthoracic echocardiography demonstrated normal biventricular function with LVEF $>55 \%$, grade I diastolic dysfunction, and without valvular abnormality. Initial ECG showed normal sinus rhythm with a heart rate of $85 \mathrm{bpm}$, left axis deviation, left ventricular hypertrophy, and age undetermined anterior infarct (Fig. 2a). Her chest X-ray showed bilateral opacities consistent with COVID-19 pneumonia which was treated conservatively. On the eighth day of admission, her telemetry strips demonstrated sinus arrest with substantial pauses of up to $5.3 \mathrm{~s}$ (Fig. 2b). In addition, she had a short run of non-sustained ventricular tachycardia (NSVT) and up to $10 \mathrm{~min}$ of a supraventricular tachycardia (SVT) that terminated spontaneously (Fig. 2b). She remained asymptomatic during all arrhythmic episodes. For blood pressure management prior to admission, she was on metoprolol succinate $25 \mathrm{mg}$ dose without any episodes of bradycardia or pauses; however, it was discontinued during this admission due to concern for sinus node dysfunction. Throughout hospitalization, her respiratory status remained stable. Her conduction system gradually improved with shorter sinus pauses lasting $2-3$ s. Ultimately, by day 10 , no sinus pauses were reported, and by day 14, no further arrhythmias had recurred (Fig. 2c).

Differential diagnosis for this case is similar to patient 1. Mildly elevated troponin that peaked at $0.92 \mathrm{ng} / \mathrm{mL}$ can be 
a

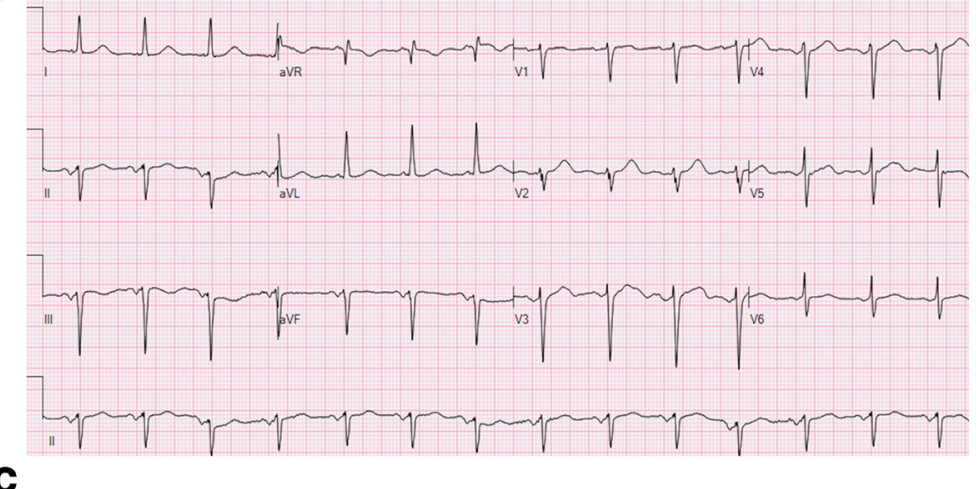

C

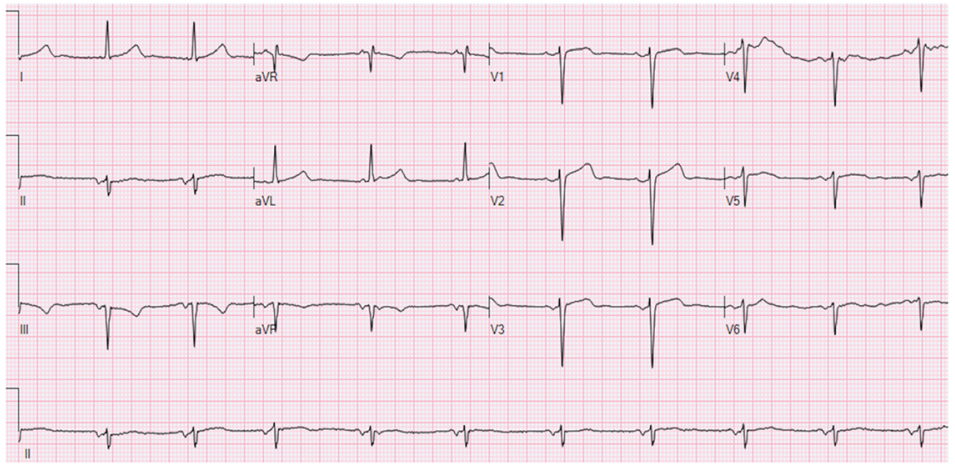

b

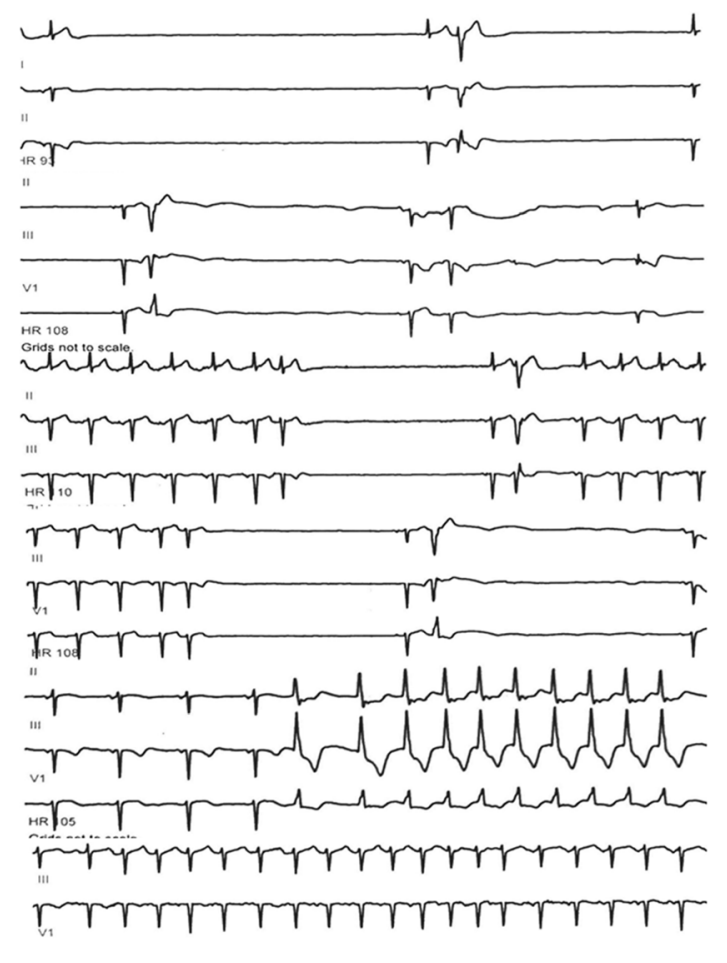

Fig. 2 Representative electrocardiogram and telemetry tracings of the patient in case 2. a Electrocardiogram demonstrating normal sinus rhythm heart rate of $85 \mathrm{bpm}$, left axis deviation, left ventricular hypertrophy, and age undetermined anterior infarct. b Telemetry tracings of the patient demonstrating sinus arrest with substantial pauses in the top 4 tracings. Non-sustained monomorphic tachycardia and sinus tachycardia in the bottom 2 tracings. c Electrocardiogram after the episodes of pause and arrest demonstrating normal sinus rhythm with heart rate of $63 \mathrm{bpm}$, left axis deviation, and age undetermined anterior infarct attributed to supply demand mismatch in the setting of acute SARS-CoV2 infection and uncontrolled hypertension.

\section{Discussion}

Recent studies have suggested that myocardial injury is common especially in critically ill COVID-19 patients through different mechanisms mainly due to direct damage to the cardiomyocytes and systemic inflammation [1]. Furthermore, cardiac troponin elevation was observed in COVID-19 patients without underlying cardiovascular disease [1]. Cardiac conduction system disease involving the SA node and AV node has been shown to be caused by various infections including viral myocarditis and severe acute respiratory syndrome $[2,5,6]$. Whether the underlying mechanism of cardiac arrhythmias in COVID-19 is a result of myocardial and/or conduction system damage, or the virus has any direct arrhythmogenic effect is not yet established. We report two cases with COVID-19 and cardiac conduction system abnormalities. These cases were specifically selected because despite reported histories of normal conduction systems and lack of nodal blocking agents along with normal initial tests including ECGs, electrolytes, and echocardiography, these patients developed bradyarrhythmias. Additionally, their bradyarrhythmias improved without intervention as these patients recovered from initial COVID-19 insults, suggesting highly the correlation of the bradyarrhythmias to SARSCoV2 infection. SARS-CoV-2 can cause reversible cardiac conduction disease in stable patients without underlying electrophysiologic cardiac disease. However, short-term mortality and morbidity is high in patients with COVID-19 and severe bradycardia requiring pacing support. This emphasizes the importance of bradyarrhythmias in the prognosis of COVID19 patients [7].

\section{Conclusions}

The potential mechanisms for arrhythmia include myocardial inflammation leading to nodal or conduction system dysfunction or direct viral damage [2]. Patients diagnosed with COVID-19 infection should be monitored closely for the development of bradyarrhythmia. Limitation of these case reports is lack of the cardiac magnetic resonance with contrast to evaluate inflammation and/or myocarditis. 
Acknowledgments The authors are grateful to our colleagues, who contributed invaluable clinical information.

\section{Compliance with Ethical Standards}

Conflict of Interest The authors declare that they have no conflicts of interest.

Ethical Approval Research conducted according to ethical guidelines.

Consent Written informed consent was obtained.

\section{References}

1. Babapoor-Farrokhran S, Gill D, Walker J, Rasekhi RT, Bozorgnia B, Amanullah A. Myocardial injury and COVID-19: possible mechanisms. Life Sci. 2020;28:117723. https://doi.org/10.1016/j.lfs.2020. 117723 .
2. Babapoor-Farrokhran S, Rasekhi RT, Gill D, Babapoor S, Amanullah A. Arrhythmia in COVID-19. SN Compr Clin Med. 2020;14:1-6.

3. Liu K, Fang YY, Deng Y, Liu W, Wang MF, Ma JP, Xiao W, Wang YN, Zhong MH, Li CH, Li GC. Clinical characteristics of novel coronavirus cases in tertiary hospitals in Hubei Province. Chin Med J. 2020

4. Wang D, Hu B, Hu C, Zhu F, Liu X, Zhang J, et al. Clinical characteristics of 138 hospitalized patients with 2019 novel coronavirusinfected pneumonia in Wuhan, China. Jama. 2020;323(11):1061-9.

5. Kirmser R, Umbach R, Rowett D, Ross A. Complete heart block due to acute nonspecific carditis. Chest. 1977;71(5):682-4.

6. Yu CM, Wong RS, Wu EB, Kong SL, Wong J, Yip GW, et al. Cardiovascular complications of severe acute respiratory syndrome. Postgrad Med J. 2006;82(964):140-4.

7. Chinitz JS, Goyal R, Harding M, Veseli G, Gruberg L, Jadonath R, et al. Brady-arrhythmias in patients with COVID-19: marker of poor prognosis? Pacing Clin Electrophysiol.

Publisher's Note Springer Nature remains neutral with regard to jurisdictional claims in published maps and institutional affiliations. 